Canadian University Music Review

Canadian University Music Review

Revue de musique des universités canadiennes

Ramón Pelinski, édit., Tango nomade : études sur le tango

transculturel. Montréal : Tryptique, 1995. 469 p. ISBN

2-89031-227-5. Glossaire, bibliographie, discographie, index.

Collaborateurs : Enrique Cámara de Landa, Xavier Febrés,

Patrícia Gabancho, Béatrice Humbert, Shuhei Hokosawa, Jorge

Luis Lombardero Menéndez, Pierre Monette, Mario Paoletti,

Ramón Pelinski, William Schimmel, Pablo Vila et Susana

Weich-Shahak

\title{
Marielle Leroux
}

Volume 19, numéro 1, 1998

URI : https://id.erudit.org/iderudit/1014617ar

DOI : https://doi.org/10.7202/1014617ar

Aller au sommaire du numéro

Éditeur(s)

Canadian University Music Society / Société de musique des universités canadiennes

ISSN

0710-0353 (imprimé)

2291-2436 (numérique)

Découvrir la revue

Citer ce compte rendu

Leroux, M. (1998). Compte rendu de [Ramón Pelinski, édit., Tango nomade : études sur le tango transculturel. Montréal : Tryptique, 1995. 469 p. ISBN 2-89031-227-5. Glossaire, bibliographie, discographie, index. Collaborateurs : Enrique Cámara de Landa, Xavier Febrés, Patrícia Gabancho, Béatrice Humbert, Shuhei Hokosawa, Jorge Luis Lombardero Menéndez, Pierre Monette, Mario Paoletti, Ramón Pelinski, William Schimmel, Pablo Vila et Susana Weich-Shahak]. Canadian University Music Review / Revue de musique des universités canadiennes, 19(1), 116-118. https://doi.org/10.7202/1014617ar

All Rights Reserved @ Canadian University Music Society / Société de musique des universités canadiennes, 1998
Ce document est protégé par la loi sur le droit d'auteur. L’utilisation des services d'Érudit (y compris la reproduction) est assujettie à sa politique d'utilisation que vous pouvez consulter en ligne.

https://apropos.erudit.org/fr/usagers/politique-dutilisation/ 
l'absence d'écrits importants, notamment ceux de Mario Bois, de Maurice Fleuret et de Thomas de $\mathrm{Lio}^{8}$.

La présence des références bibliographiques, des encadrés et des notes n'est mentionnée que dans le premier encadré explicatif (p. 12). Cela gêne quelque peu le lecteur qui doit sans cesse revenir en arrière pour retrouver la note ou l'encadré correspondant à la référence en question. L'auteur aurait pu tout simplement utiliser l'appel de note à côté du titre de l'encadré et mettre la référence bibliographique en note infrapaginale. Les références relatives aux notes explicatives auraient pu être tout simplement ajoutées à la suite.

Delalande a su poser des questions pertinentes afin de découvrir l'essentiel sur Xenakis comme compositeur, et ce, $d$ 'une manière.remarquablement accessible. Il s'agit d'un ouvrage digne de figurer dans une bibliothèque qui se veut au fait des développements les plus récents, bien qu'une grande partie de son contenu date de plus d'une décennie. Qu'il se destine à la composition ou à l'interprétation, l'étudiant en musique qui veut s'initier à l'œuvre de Xenakis y trouvera certainement son compte. Le musicologue devra toutefois considérer cet ouvrage comme complémentaire, en raison des faiblesses mineures que nous avons signalées.

Paulin Daigle

Ramón Pelinski, édit., Tango nomade : études sur le tango transculturel. Montréal : Tryptique, 1995. 469 p. ISBN 2-89031-227-5. Glossaire, bibliographie, discographie, index. Collaborateurs : Enrique Cámara de Landa, Xavier Febrés, Patrícia Gabancho, Béatrice Humbert, Shuhei Hokosawa, Jorge Luis Lombardero Menéndez, Pierre Monette, Mario Paoletti, Ramón Pelinski, William Schimmel, Pablo Vila et Susana Weich-Shahak.

Tango, tanguero, tanguera, tangologie, tanguistique et tanguidité. Le monde du tango est peut-être plus vaste et plus complexe qu'on pourrait le penser. Au début des années 80, Ramón Pelinski était invité par Irma Ruiz, de l'Institut national de musicologie de Carlos-Vega de Buenos Aires, à rédiger, pour le second volume de l'Antología del tango rioplatense, la partie portant sur le tango argentin à l'étranger. Jusqu'à récemment professeur de musicologie à l'Université de Montréal, Pelinski est le fondateur du premier ensemble de tango argentin du Canada, dont il a été le pianiste et l'arrangeur; il est également l'auteur d'études sur le tango publiées dans Latin American Music Review (1989), Popular Music Perspectives (1985) et Études françaises (1981). Ses recherches l'ont conduit à la publication de Tango nomade, un ouvrage qui réunit les réflexions et les recherches d'un ensemble de tangologues cuvrant dans divers pays. Tango nomade ne prétend pas dresser une liste

des premières œuvres (1953-69) de I. Xenakis : pour une approche historique de l'émergence du phenomène du son » (thèse de doctorat, Université de Paris IV, 1993).

8Mario Bois, Iannis Xenakis : The Man and His Music (Westport, Conn. : Greenwood Press, 1980); Maurice Fleuret, Xenakis (Paris : Salabert, 1978); Thomas DeLio, « Iannis Xenakis's Nomos Alpha: The Dialectics of Structure and Materials », Journal of Music Theory 24 (1980) : 63-96. 
exhaustive de tous les endroits où se retrouve le tango, les auteurs cherchant plutôt à explorer comment il se transforme au contact de différentes populations et à déterminer les attributs qui lui permettent de séduire différents peuples.

Le tango est né à la fin du $\mathrm{XIX}^{\mathrm{e}}$ siècle, dans les proches environs de Buenos Aires. Il provient des milieux pauvres où se côtoient natifs et immigrants, des milieux en relation avec le crime et la prostitution. Il est un produit d'un métissage culturel et n'a cessé de voyager et de se renouveler au contact de nouvelles cultures. Le texte de Pablo Vila, "Le tango et la formation des identités ethniques en Argentine ", raconte les origines du tango et son développement au sein de son pays natal.

Bientôt, le tango voyage hors de sa terre d'origine et on le retrouvera un peu partout autour du monde; citons, entre autres, Paris, Rome, Barcelone, Helsinki, Tokyo, Montréal et New York. Au contact de nouvelles cultures, il se transforme et influence à son tour ses terres d'accueil. Par exemple, en entrant dans les salons parisiens, au début du $\mathrm{XX}^{\mathrm{e}}$ siècle, la nouvelle danse provoque autant d'engouement que de condamnations, défenseurs et détracteurs s'affrontent (voir Béatrice Humbert, «Le tango à Paris de 1907 à 1920 »). Dans la ville de Seinäjoki, dans l'ouest de la Finlande, l'arrivée de l'été est synonyme de l'arrivée du tango. La ville est l'hôte d'un très important festival de tango qui réunit chanteurs, danseurs, paroliers et compositeurs de tango (Jorge Lombardo, « Notes sur le tango en Finlande et dans les pays nordiques »).

Dans son texte "Tango nomade ", Ramón Pelinski distingue entre deux types « idéaux » de tango : le tango porteño, territorialisé sur les rives du Río de la Plata, et plus particulièrement à Buenos Aires, et le tango nomade, qui s'est reterritorialisé à l'étranger. L'auteur propose un cadre théorique servant à l'interprétation du nomadisme du tango et esquisse un panorama général de sa transculturation. Ce cadre théorique offre un bon point de départ qui nous aide à faire le lien entre les divers textes présentés. On pourrait aussi le lire en dernier, en guise de conclusion à ce voyage autour du monde sur le thème du tango. L'article de Pelinski est le plus théorique du recueil et, bien qu'il soit clairement présenté, il est probablement également le moins facile à lire, peut-être à cause du (trop?) grand nombre de mots composés avec préfixes : alloculturel, autodifférentielle, coprésence, ethnocentrisme, interculturel, transculturation, transnational, transrégionalisé, réinterprétation, etc.

La lecture de Tango nomade sensibilise sur la place occupée par ce genre autant dans la musique dite savante, dans la musique populaire que dans les milieux de la danse. On note que ce sont parfois la musique, parfois la chorégraphie, ou parfois encore les paroles véhiculées par le tango qui gagnent la faveur de ses nouveaux adeptes. Le lecteur québécois, et plus particulièrement montréalais, saura découvrir où se trouvent les traces du tango au sein de sa propre culture dans « Série tango : le milieu du tango à Montréal » de Pierre Monette. Soulignons ici l'excellent travail de traduction et d'établissement du glossaire de ce dernier. 
En parlant de tango, on ne peut passer sous silence certains grands noms qui ont joué un rôle majeur dans son histoire. Ainsi on retrouve des textes consacrés à Astor Piazzolla, compositeur qui donne une nouvelle dimension au tango et qui l'incorpore à la musique de tradition savante; à Jorge Luis Borges, écrivain qui ouvre au tango la porte de son œuvre poétique et littéraire; et à l'acteur et chanteur Carlos Gardel, sans doute le plus célèbre chanteur de tango.

L'ouvrage contient plusieurs illustrations qui complètent les textes. On n'y trouve cependant que peu d'exemples musicaux, à l'exception de l'article « Le tango séfarade » de Susana Weich-Shahak. Cette dernière traite de l'intégration du tango en tant que genre vocal dans le répertoire séfarade.

Tango nomade abonde en informations sur l'histoire du tango à travers les pays et les cultures. De nombreuses anecdotes colorent les articles et incitent à poursuivre la lecture. Il intéressera bien sûr les personnes qui aiment le tango, mais également celles qui se penchent sur l'étude des transformations et des migrations des musiques ou des danses à caractère national. Pour compléter la lecture de Tango nomade, on suggérera l'audition de quelques tangos; la discographie contenue dans le volume pourra fournir des titres.

Marielle Leroux

André-Marie Despringre, édit. Chants enfantins d'Europe : systèmes poéticomusicaux de jeux chantés (France, Espagne, Chypre, Italie). Paris : Éditions l'Harmattan, 1997. 215 p. ISBN 2- 7384-6054-2.

André-Marie Despringre et Andy Arleo, « Musilinguistique du chant enfantin »; Andy Arleo, «Un jeu de dominos verbal »; Jeanine Fribourg et André-Marie Despringre, «Jeux chantés en Espagne »; Photini Panayi, « La poéticité dans l'élimination : analyse linguistique et musicale de formulettes chypriotes »; Emmanuelle Olivier, «D'un répertoire enfantin et de ses diverses formes poético-musicales ».

Le livre Chants enfantins d'Europe rend compte d'une recherche réalisée par un groupe d'ethnomusicologues et d'ethnolinguistes identifié sous le nom original et descriptif de Musilingue, collectif créé en 1988 au Laboratoire de Langues et Civilisations à Tradition Orale (LACITO-CNRS) et qui centre ses recherches sur les relations existant entre la musique et la langue dans le domaine de la poésie chantée de tradition orale. Il s'agit d'une étude comparative de chants d'enfants de Chypre, d'Espagne, de France et d'Italie qui cherche à mettre en évidence les interactions entre les différents niveaux de jeux chantés (mélodie, rythme, gestes, textes, etc.) et leurs particularités culturelles observées dans des situations d'acquisition. Cette nouvelle parution s'inscrit dans le prolongement d'une étude diffusée en 1991 sous le titre de Poésies chantées de tradition orale en Flandre et en Bretagne (Paris : Champion). L'ouvrage compte six chapitres encadrés d'un avant-propos rédigé par le directeur de la publication, André-Marie Despringre, et d'une bibliographie générale. Jean Molino signe la préface.

C'est la musilinguistique du chant enfantin - concept présenté par les auteurs Andy Arleo et André-Marie Despringre - qui est examiné dans le 